\title{
Study of the 1D Saint-Venant Equations and Application to the Simulation of a Flood Problem
}

\author{
Maman Yarodji Abdoul Kader, Rabé Badé, Bisso Saley \\ Department of Mathematics and Computer Science, Faculty of Science and Technology, Abdou Moumouni University, \\ Niamey, Niger \\ Email: yalokmaman@gmail.com, baderabe@yahoo.fr, bsaley@yahoo.fr
}

How to cite this paper: Kader, M.Y.A., Badé, R. and Saley, B. (2020) Study of the 1D Saint-Venant Equations and Application to the Simulation of a Flood Problem. Journal of Applied Mathematics and Physics, 8, 1193-1206.

https://doi.org/10.4236/jamp.2020.87090

Received: May 9, 2020

Accepted: July 5, 2020

Published: July 8, 2020

Copyright $\odot 2020$ by author(s) and Scientific Research Publishing Inc. This work is licensed under the Creative Commons Attribution International License (CC BY 4.0).

http://creativecommons.org/licenses/by/4.0/

\begin{abstract}
In this paper we are concerned with the mathematical and numerical analysis of the one-dimensional Saint-Venant equations. Thus, we prove the existence of a weak solution for any fixed time and with low regularity on the data. For the numerical approach we use the Rusanov scheme to approximate the flux and the hydrostatic reconstruction method which consists of decentering the source term at the interface. A numerical test of the proposed resolution is performed on a non-uniform topography.
\end{abstract}

\section{Keywords}

Shallow Water Equations, Hyperbolic System, Finite Volume Method, Weak Solution

\section{Introduction}

The Saint-Venant equations are very used to describe many physical phenomena: like the runoff [1] [2], the transport of pollutants in the river [3] [4]. These equations are based on certain physical laws, namely the mass conservation and the momentum conservation [5]. Due to the recurrence of floods around the world and in particular in arid countries, we use these equations to study this phenomenon of floods.

Inspired by the hyperbolic nature of the Saint-Venant equations, we establish an existence result of weak solution using the Rankine-Hugoniot relation. To do this, we subdivide the domain into two parts and we first prove the existence on each part. Then, the existence in the whole domain is obtained if we have at the interface separating the two subdomains: the equality between the jump of the 
continuous flux and the jump of conservative variables.

The paper is organised as follows. In Section 2 we make a brief presentation of the Saint-Venant equations. Section 3 is devoted to showing the existence of a weak solution of Saint-Venant equations. In Section 4, the numerical solution is computed by the finite volume methods where the discrete flux is obtained by the Rusanov scheme and the topography term is processed by the hydrostatic reconstruction method combined with the well-balanced scheme. This scheme preserves the positivity of the height. Finally, Section 5 is intended for the numerical simulation of the flood phenomenon on a domain with not completely flat topography.

\section{Continuous Problem}

The Saint-Venant equations derive from the Navier-Stokes equations by performing an average operation along the vertical, the hydrostatic and the impermeability assumptions [6]. The mathematical model relates the fluid height $h$ and the flow velocity $u$. In one-dimensional they are given by:

$$
\left\{\begin{array}{l}
\frac{\partial h}{\partial t}+\frac{\partial h u}{\partial x}=0 \\
\frac{\partial h u}{\partial t}+\frac{\partial}{\partial x}\left(h u^{2}+\frac{g h^{2}}{2}\right)=-g h \frac{\partial z}{\partial x}
\end{array}\right.
$$

where $g$ is the gravity, $z$ the bottom topography. This hyperbolic system can be rewritten into the form:

$$
\frac{\partial \boldsymbol{U}}{\partial t}(x, t)+\frac{\partial(\boldsymbol{f}(\boldsymbol{U}))}{\partial x}(x, t)=\boldsymbol{S},
$$

with $\boldsymbol{U}=(h, q), \quad \boldsymbol{f}(\boldsymbol{U})=\left(\begin{array}{c}q \\ \frac{q^{2}}{h}+\frac{g h^{2}}{2}\end{array}\right), \quad \boldsymbol{S}=\left(\begin{array}{c}0 \\ -g h \frac{\partial z}{\partial x}\end{array}\right)$ and $q=h u$ the momentum.

\section{Weak Solution of the Saint-Venant 1D System}

The system (2) being hyperbolic, we will show the existence of a weak solution of the Saint-Venant equations with $S=0$. For this aim we use the mathematical analysis of hyperbolic partials differentials equations [7]:

$$
\begin{cases}\frac{\partial \boldsymbol{U}}{\partial t}(x, t)+\frac{\partial(\boldsymbol{f}(\boldsymbol{U}))}{\partial x}(x, t)=0, & (x, t) \in \mathbb{R} \times \mathbb{R}_{+}^{*} \\ \boldsymbol{U}(x, 0)=\boldsymbol{U}_{0}(x) & \forall x \in \mathbb{R}\end{cases}
$$

with Dirichlet boundary conditions.

Definition 1. Let $\boldsymbol{U}_{0} \in L^{\infty}(\mathbb{R})^{2}$ and $\boldsymbol{f} \in C^{1}(\mathbb{R})^{2}$.

$A$ weak solution of $(3)$ is a function $\boldsymbol{U} \in L^{\infty}\left(\mathbb{R} \times \mathbb{R}_{+}\right)^{2}$ such as:

$$
\int_{\mathbb{R}^{\prime} \mathbb{R}_{+}}\left(\boldsymbol{U}(x, t) \varphi_{t}(x, t)+\boldsymbol{f}(\boldsymbol{U})(x, t) \varphi_{x}(x, t)\right) \mathrm{d} x \mathrm{~d} t+\int_{\mathbb{R}} \boldsymbol{U}(x, 0) \varphi(x, 0) \mathrm{d} x=0
$$

for all $\varphi$ a continuous derivable function with compact support on $\mathbb{R} \times \mathbb{R}_{+}$ 
$\left(\forall \varphi \in C_{c}^{1}\left(\mathbb{R} \times \mathbb{R}_{+}\right)\right)$.

Proposition 1. Let $D$ be an operator in time or space, then we have:

$$
D \int_{z_{f}}^{z_{s}} F \mathrm{~d} z=\int_{z_{f}}^{z_{s}} D F \mathrm{~d} z+F\left(x, y, z_{s}\right) D z_{s}-F\left(x, y, z_{f}\right) D z_{f}
$$

Theorem 2. Let assume the curve $\Gamma_{t}^{x}=\left\{(x, t) \in \mathbb{R} \times \mathbb{R}_{+}^{*}, x=\sigma(t)\right\}$ where $\sigma \in C^{1}\left(\mathbb{R}_{+}\right) . \Gamma_{t}^{x}$ partitions an open domain $\Omega \subset \mathbb{R} \times \mathbb{R}_{+}$into two subdomains: $\Omega_{-}$and $\Omega_{+}$, given by:

$$
\Omega_{-}=\left\{(x, t) \in \Omega, x<\Gamma_{t}^{x}\right\}, \Omega_{+}=\left\{(x, t) \in \Omega, x>\Gamma_{t}^{x}\right\} .
$$

A function $U \in C^{1}\left(\Omega_{-}\right)^{2} \cup C^{1}\left(\Omega_{+}\right)^{2}$ is a weak solution of $(3)$ if and only if

$$
-\sigma^{\prime}\left(\boldsymbol{U}^{+}-\boldsymbol{U}^{-}\right)+\left(\boldsymbol{f}\left(\boldsymbol{U}^{+}\right)-\boldsymbol{f}\left(\boldsymbol{U}^{-}\right)\right)=0,
$$

where $\boldsymbol{U}^{+}(t)=\lim _{x \rightarrow \sigma t^{+}} \boldsymbol{U}(x, t)$ and $\boldsymbol{U}^{-}(t)=\lim _{x \rightarrow \sigma t^{-}} \boldsymbol{U}(x, t)$.

Proof. Let $\boldsymbol{U}^{\mathrm{x} \rightarrow \sigma t^{+}}$be a weak solution of the problem (2) with $\boldsymbol{S}=0$, then we have:

$$
\int_{{\mathbb{R} \times \mathbb{R}_{+}}}\left(\boldsymbol{U}(x, t) \varphi_{t}(x, t)+\boldsymbol{f}(\boldsymbol{U})(x, t) \varphi_{x}(x, t)\right) \mathrm{d} x \mathrm{~d} t+\int_{\mathbb{R}} \boldsymbol{U}(x, 0) \varphi(x, 0) \mathrm{d} x=0
$$

$\forall \varphi \in C_{c}^{1}\left(\mathbb{R} \times \mathbb{R}_{+}\right)$. By choosing $\varphi$ such as $\operatorname{supp}(\varphi) \subset \Omega_{+}$, we obtain:

$$
\int_{\mathbb{R}^{\prime} \mathbb{R}_{+}}\left(\boldsymbol{U}(x, t) \varphi_{t}(x, t)+\boldsymbol{f}(\boldsymbol{U})(x, t) \varphi_{x}(x, t)\right) \mathrm{d} x \mathrm{~d} t=0
$$

$\forall \varphi \in C_{c}^{1}\left(\mathbb{R} \times \mathbb{R}_{+}\right)$. An integration by part in $\Omega_{+}$, giving:

$$
\int_{\Omega_{+}}\left(\boldsymbol{U}_{t}(x, t)+(\boldsymbol{f}(\boldsymbol{U}))_{x}\right) \varphi(x, t) \mathrm{d} x \mathrm{~d} t=0, \quad \forall \varphi \in C_{c}^{1}\left(\mathbb{R} \times \mathbb{R}_{+}\right)
$$

$\varphi$ being arbitrary we deduce:

$$
\boldsymbol{U}_{t}(x, t)+(\boldsymbol{f}(\boldsymbol{U}))_{x}=0 .
$$

This proves that $U$ is solution in $\Omega_{+}$.

Similarly we prove that $\boldsymbol{U}$ is solution in $\Omega_{-}$.

Let us now consider: $M_{1}, M_{2}$ in $\mathbb{R} ; t_{1}, t_{2}$ in $\mathbb{R}_{+}$and $\varphi$ such as

$$
\begin{gathered}
\operatorname{supp}(\varphi) \subset] M_{1}, M_{2}[\times] t_{1}, t_{2}[ \\
\int_{{\mathbb{R} \times \mathbb{R}_{+}}}\left(\boldsymbol{U}(x, t) \varphi_{t}(x, t)+\boldsymbol{f}(\boldsymbol{U})(x, t) \varphi_{x}(x, t)\right) \mathrm{d} x \mathrm{~d} t=0 .
\end{gathered}
$$

Performing the integration on $] M_{1}, M_{2}[\times] t_{1}, t_{2}[$, we have:

$$
\begin{aligned}
& \int_{t_{1}}^{t_{2}} \int_{M_{1}}^{M_{2}}\left(\boldsymbol{U}(x, t) \varphi_{t}(x, t)+\boldsymbol{f}(\boldsymbol{U})(x, t) \varphi_{x}(x, t)\right) \mathrm{d} x \mathrm{~d} t=0, \\
& \int_{t_{1}}^{t_{2}} \int_{\sigma(t)}^{M_{2}}\left(\boldsymbol{U}(x, t) \varphi_{t}(x, t)+\boldsymbol{f}(\boldsymbol{U})(x, t) \varphi_{x}(x, t)\right) \mathrm{d} x \mathrm{~d} t \\
& +\int_{t_{1}}^{t_{2}} \int_{M_{1}}^{\sigma(t)}\left(\boldsymbol{U}(x, t) \varphi_{t}(x, t)+\boldsymbol{f}(\boldsymbol{U})(x, t) \varphi_{x}(x, t)\right) \mathrm{d} x \mathrm{~d} t=0 .
\end{aligned}
$$

Let us set:

$$
\begin{aligned}
& I=\int_{t_{1}}^{t_{2}} \int_{\sigma(t)}^{M_{2}}\left(\boldsymbol{U}(x, t) \varphi_{t}(x, t)+\boldsymbol{f}(\boldsymbol{U})(x, t) \varphi_{x}(x, t)\right) \mathrm{d} x \mathrm{~d} t, \\
& J=\int_{t_{1}}^{t_{2}} \int_{M_{1}}^{\sigma(t)}\left(\boldsymbol{U}(x, t) \varphi_{t}(x, t)+\boldsymbol{f}(\boldsymbol{U})(x, t) \varphi_{x}(x, t)\right) \mathrm{d} x \mathrm{~d} t .
\end{aligned}
$$

We begin with $I$ 


$$
\begin{aligned}
I= & \int_{t_{1}}^{t_{2}} \int_{\sigma(t)}^{M_{2}} \boldsymbol{U}(x, t) \varphi_{t}(x, t) \mathrm{d} x \mathrm{~d} t+\int_{t_{1}}^{t_{2}} \int_{\sigma(t)}^{M_{2}} \boldsymbol{f}(\boldsymbol{U})(x, t) \varphi_{x}(x, t) \mathrm{d} x \mathrm{~d} t \\
= & \int_{t_{1}}^{t_{2}} \int_{\sigma(t)}^{M_{2}}\left((\boldsymbol{U} \varphi)_{t}-\boldsymbol{U}_{t} \varphi\right)(x, t) \mathrm{d} x \mathrm{~d} t-\int_{t_{1}}^{t_{2}} \boldsymbol{f}(\boldsymbol{U})\left(\sigma^{+}(t), t\right) \varphi(x, t) \mathrm{d} t \\
& -\int_{t_{1}}^{t_{2}} \int_{\sigma(t)}^{M_{2}}(\boldsymbol{f}(\boldsymbol{U}))_{x}(x, t) \varphi(x, t) \mathrm{d} x \mathrm{~d} t .
\end{aligned}
$$

So by Proposition 1, with $D=\frac{\mathrm{d}}{\mathrm{d} t}$ we get:

$$
\begin{aligned}
I= & \int_{t_{1}}^{t_{2}}\left[\frac{\mathrm{d}}{\mathrm{d} t}\left(\int_{\sigma(t)}^{M_{2}} \boldsymbol{U}(x, t) \varphi(x, t) \mathrm{d} x\right)+\sigma^{\prime}(t) \boldsymbol{U}\left(\sigma^{+}(t), t\right) \varphi(\sigma(t), t)\right] \mathrm{d} t \\
& -\int_{t_{1}}^{t_{2}} \int_{\sigma(t)}^{M_{2}} \boldsymbol{U}_{t}(x, t) \varphi(x, t) \mathrm{d} x \mathrm{~d} t-\int_{t_{1}}^{t_{2}} \boldsymbol{f}(\boldsymbol{U})\left(\sigma^{+}(t), t\right) \varphi(x, t) \mathrm{d} t \\
& -\int_{t_{1}}^{t_{2}} \int_{\sigma(t)}^{M_{2}}(\boldsymbol{f}(\boldsymbol{U}))_{x}(x, t) \varphi(x, t) \mathrm{d} x \mathrm{~d} t \\
= & \int_{t_{1}}^{t_{2}} \sigma^{\prime}(t) \boldsymbol{U}\left(\sigma^{+}(t), t\right) \varphi(\sigma(t), t) \mathrm{d} t-\int_{t_{1}}^{t_{2}} \boldsymbol{f}(\boldsymbol{U})\left(\sigma^{+}(t), t\right) \varphi(\sigma(t), t) \mathrm{d} t \\
& -\int_{t_{1}}^{t_{2}} \int_{\sigma(t)}^{M_{2}}\left(\boldsymbol{U}_{t}(x, t)+(\boldsymbol{f}(\boldsymbol{U}))_{x}(x, t)\right) \varphi(x, t) \mathrm{d} x \mathrm{~d} t .
\end{aligned}
$$

Finally we obtain:

$$
I=-\int_{t_{1}}^{t_{2}}\left(\boldsymbol{f}(\boldsymbol{U})\left(\sigma^{+}(t), t\right)-\sigma^{\prime}(t) \boldsymbol{U}\left(\sigma^{+}(t), t\right)\right) \varphi(\sigma(t), t) \mathrm{d} t .
$$

By the same calculations we have:

$$
J=\int_{t_{1}}^{t_{2}}\left(\boldsymbol{f}(\boldsymbol{U})\left(\sigma^{-}(t), t\right)-\sigma^{\prime}(t) \boldsymbol{U}\left(\sigma^{-}(t), t\right)\right) \varphi(\sigma(t), t) \mathrm{d} t .
$$

The summation of $I$ and $J$ implies:

$$
\left(\boldsymbol{f}\left(U^{+}\right)-\boldsymbol{f}\left(U^{-}\right)\right)=\sigma^{\prime}\left(\boldsymbol{U}^{+}-\boldsymbol{U}^{-}\right) .
$$

For the reciprocal, it is sufficient to do the same calculations in the opposite direction.

\section{Numerical Resolution}

The numerical discretization of the system (2) is done by using the finite volume method [8], so let us consider a finite volume mesh: with mean a union of disjoint intervals $\left.C_{i}=\right]_{i-\frac{1}{2}} ; x_{i+\frac{1}{2}}\left[\right.$ of size $\left|C_{i}\right|=\Delta x$, centered at $x_{i}=\frac{x_{i-\frac{1}{2}}+x_{i+\frac{1}{2}}}{2}$, $i=1, \cdots, N$. Let us denote $\Delta t$ the time stepsize and $u_{i}^{n}$ the approximation of the cell average of the exact solution $u(t, x)$ at time $t^{n}=n \Delta t$.

The finite volume method consists to integrate the system on $\left[t^{n}, t^{n+1}\right] \times C_{i}$, which gives:

$$
\boldsymbol{U}_{i}^{n+1}-\boldsymbol{U}_{i}^{n}+\frac{\Delta t}{\Delta x}\left(\mathcal{F}\left(\boldsymbol{U}_{i}^{n}, \boldsymbol{U}_{i+1}^{n}\right)-\mathcal{F}\left(\boldsymbol{U}_{i-1}^{n}, \boldsymbol{U}_{i}^{n}\right)\right)=\int_{t^{n}}^{t^{n+1}} \int_{x_{i-1 / 2}}^{x_{i+1 / 2}} \boldsymbol{S}(t, x) \mathrm{d} x \mathrm{~d} t,
$$

where

$$
\mathcal{F}\left(\boldsymbol{U}_{i}^{n}, \boldsymbol{U}_{i+1}^{n}\right)=\boldsymbol{F}_{i+1 / 2}^{n} ; \quad \mathcal{F}\left(\boldsymbol{U}_{i-1}^{n}, \boldsymbol{U}_{i}^{n}\right)=\boldsymbol{F}_{i-1 / 2}^{n}
$$

and 


$$
\boldsymbol{F}_{i+1 / 2}^{n} \simeq \frac{1}{\Delta t} \int_{t_{n}}^{t_{n+1}} \boldsymbol{F}\left(\boldsymbol{U}\left(t, x_{i+1 / 2}\right)\right) \mathrm{d} t ; \quad \boldsymbol{F}_{i-1 / 2}^{n} \simeq \frac{1}{\Delta t} \int_{t_{n}}^{t_{n+1}} \boldsymbol{F}\left(\boldsymbol{U}\left(t, x_{i-1 / 2}\right)\right) \mathrm{d} t .
$$

It remains now to compute the interfacial flux $F_{i+1 / 2}^{n}, F_{i-1 / 2}^{n}$ and also evaluate the discrete source term.

\subsection{Flux Approximation}

Several methods exist in literature to approximate the flux, in the present work, we use the simpler and thus more popular: the Rusanov flux [9]. It is a generalization of Lax-Friedrich flux [10]. Let us note $\boldsymbol{U}_{L}$ and $\boldsymbol{U}_{R}$ the data in the two neighborring cells, then the Rusanov flux is given by:

$$
\mathcal{F}\left(\boldsymbol{U}_{G}, \boldsymbol{U}_{D}\right)=\frac{\boldsymbol{F}\left(\boldsymbol{U}_{G}\right)+\boldsymbol{F}\left(\boldsymbol{U}_{D}\right)}{2}-c \frac{\boldsymbol{U}_{D}-\boldsymbol{U}_{G}}{2}
$$

where the Rusanov speed $c$ is given by:

$$
c=\max _{k \in\{1 ; 2\}}\left(\left|\lambda_{k}\left(\boldsymbol{U}_{G}\right)\right|,\left|\lambda_{k}\left(\boldsymbol{U}_{D}\right)\right|\right)
$$

and $\lambda_{k}$ are eigenvalues of the matrix:

$$
\frac{\partial \boldsymbol{F}}{\partial \boldsymbol{U}}=\left(\begin{array}{cc}
0 & 1 \\
g h-u^{2} & 2 u
\end{array}\right),
$$

one obtains: $\lambda_{1}(\boldsymbol{U})=u-\sqrt{g h}$ and $\lambda_{2}(\boldsymbol{U})=u+\sqrt{g h}$.

Remark 1. The time step discretization $\Delta t$, must verify the condition:

$$
c \frac{\Delta t}{\Delta x} \leq \frac{1}{2}
$$

where $c$ is the maximum wave velocity obtained by calculating the maximum eigenvalue of the local Jacobian matrix of the cell.

\subsection{Traitement of the Bottom Topography}

The well-balanced method is based on the hydrostatic reconstruction method [11] [12] [13], which consists of decentering the source term at the interfaces. These schemes have the advantage to preserve the equilibrium of the fluid, which means that the height and the velocity being constant in time. Using this property we obtain the relation:

$$
\frac{\partial}{\partial x}\left(\frac{g h^{2}}{2}\right)=-g h \frac{\partial z}{\partial x}
$$

called hydrostatic equilibrium.

Applying the differentiation rule, we show that $h+z$ is constant. The hydrostatic reconstruction method is based on the idea that near the equilibrium, the flows are almost hydrostatic.

The reconstructed water heights on either side of the interfaces adjust to satisfy equation:

$$
\frac{\partial}{\partial x}\left(\frac{g \bar{h}^{2}}{2}\right)=-g \bar{h} \frac{\partial z}{\partial x}
$$


Integrating on a cell we obtain a following discretization of the source term:

$$
-\int_{x_{i-1 / 2}}^{x_{i+1 / 2}} g \bar{h} \frac{\partial z}{\partial x}=\frac{g}{2} \bar{h}_{i+1 / 2, L}^{2}-\frac{g}{2} \bar{h}_{i-1 / 2, R}^{2},
$$

where $\bar{h}_{i+1 / 2, L}=h_{i, r}+z_{i, r}-z_{i+1 / 2}$ and $\bar{h}_{i+1 / 2, R}=h_{i+1, l}+z_{i+1, l}-z_{i+1 / 2}$ with $z_{i+1 / 2}$ is the topography at the interface gives by: $z_{i+1 / 2}=\max \left(z_{i, r} ; z_{i+1, l}\right)$.

Finally the discrete system (7) becomes:

$$
\boldsymbol{U}_{i}^{n+1}-\boldsymbol{U}_{i}^{n}+\frac{\Delta t}{\Delta x}\left(\mathcal{F}\left(\boldsymbol{U}_{i}^{n}, \boldsymbol{U}_{i+1}^{n}\right)-\mathcal{F}\left(\boldsymbol{U}_{i-1}^{n}, \boldsymbol{U}_{i}^{n}\right)\right)=\Delta t \boldsymbol{S}_{i}^{n},
$$

where $\boldsymbol{S}_{i}^{n}$ is given by:

$$
\boldsymbol{S}_{i}^{n}=\left(\begin{array}{c}
0 \\
g \frac{h_{i+1 / 2 G}^{2}}{2}-g \frac{h_{i-1 / 2 D}^{2}}{2}
\end{array}\right),
$$

with: $h_{i+1 / 2 G}=\max \left(0 ; \bar{h}_{i+1 / 2 G, r}\right) ; \quad h_{i+1 / 2 D}=\max \left(0 ; \bar{h}_{i+1 / 2 D, l}\right)$.

\section{Numerical Results}

As real numerical test application, we apply the proposed numerical resolution to observe the flow of water at the free surface on a ground having the form given in Figure 1.

The water flows from the zone (A) with an imposed discharge towards the zone (B) with a blockade at the end.

The bottom topography is given by:

$$
Z(x)= \begin{cases}0.6 & x \leq 0.25 \\ -1.6 x+1 & x \in[0.25 ; 0.5] \\ 0.2 & x \in[0.5 ; 0.7] \\ 0.2-\sqrt{0.01-(x-0.8)^{2}} & x \in[0.7 ; 0.9] \\ 0.2 & x \in[0.9 ; 1.1] \\ 0.2+\sqrt{0.01-(x-1.2)^{2}} & x \in[1.1 ; 1.3] \\ 0.2 & x \geq 1.3\end{cases}
$$

(A)

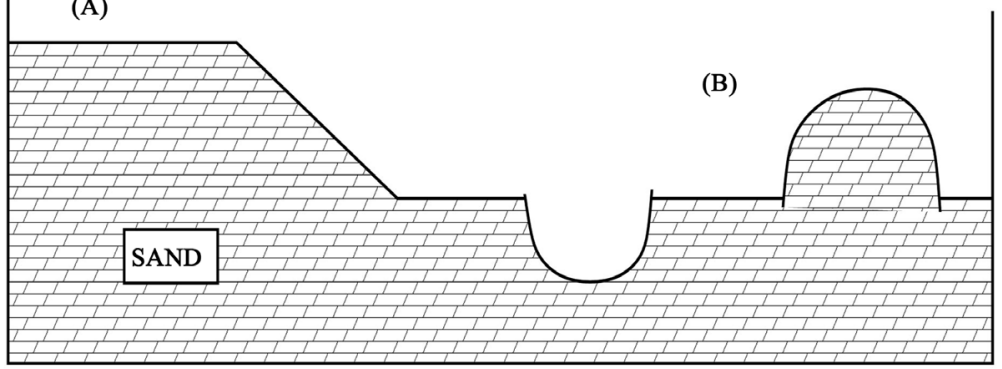

Figure 1. Form of the topography. 
with initial condition given by: $h+z=0.65 \mathrm{~m}$ and $q=0.9 \mathrm{~m}^{3} / \mathrm{s}$. The numerical tests are performed on a domain [0;1.5], subdivided into 150 cells.

The objective of this simulation is to measure the risk of flooding in urban environment with non-flat topography with a high rainfall without taking into account the rate of the infiltration. For that, we had imposed a very important flow of water upstream and a closure downstream. The question here is: how long will the water take to invade zone (B)?

In Figures 2-5: the water has submerged progressively the hump and the hollow but not completely the zone B. In Figures 6-8 we see a start of water return due to the blockade. In Figures 9-15 the water continues to flood zone B and we observe two ridges on the surface due to the meeting between the two waves: one coming from zone A and the other from zone B. In Figure 16 and Figure 17 we see that the water has completely flooded the zone B at $t=0.97 \mathrm{~s}$.

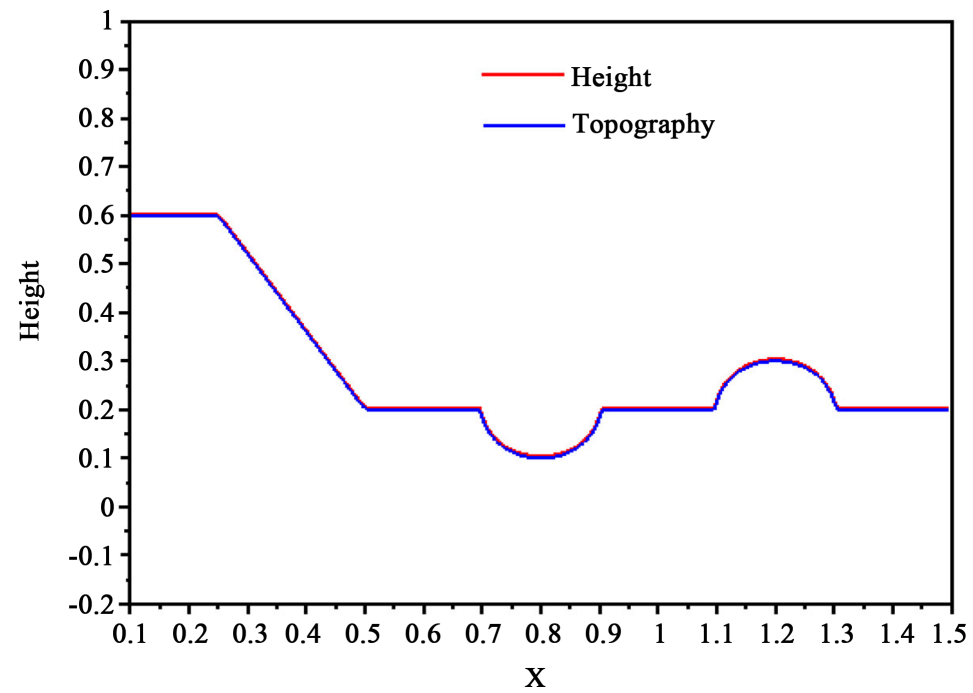

Figure 2. Water height at $t=0 \mathrm{~s}$.

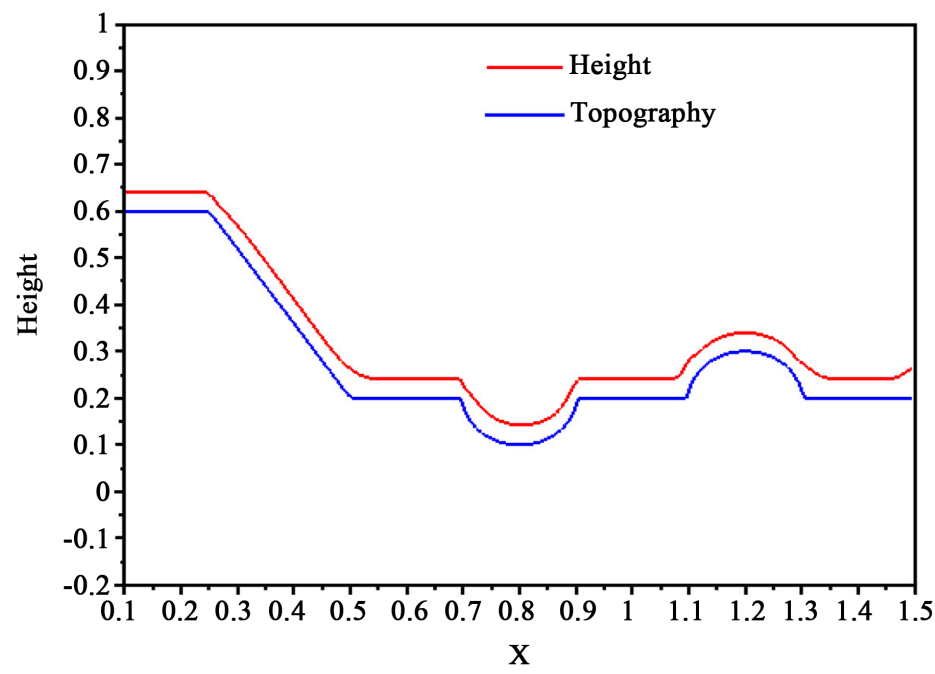

Figure 3. Water height at $t=0.0011 \mathrm{~s}$. 


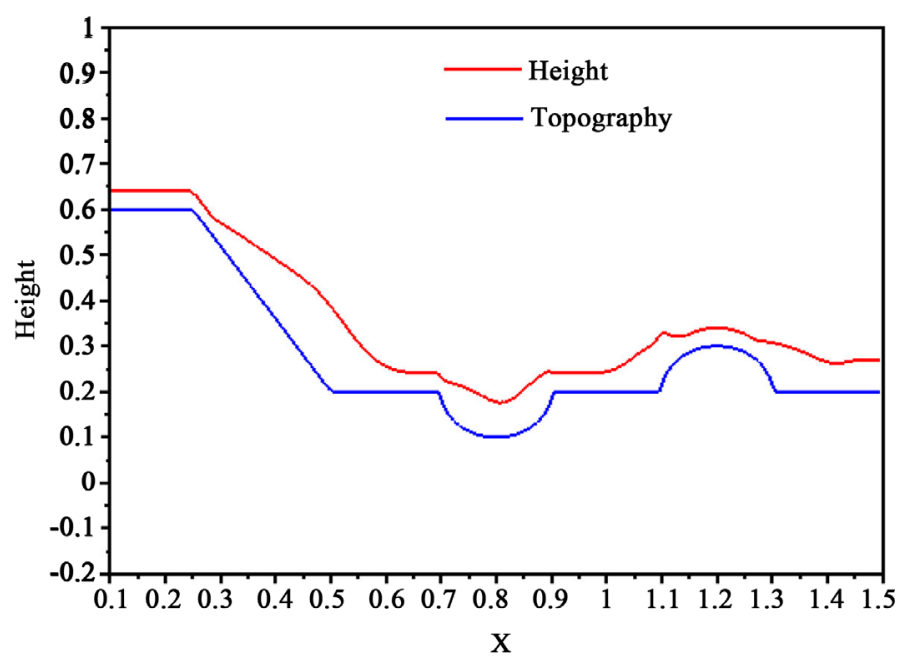

Figure 4. Water height at $t=0.012 \mathrm{~s}$.

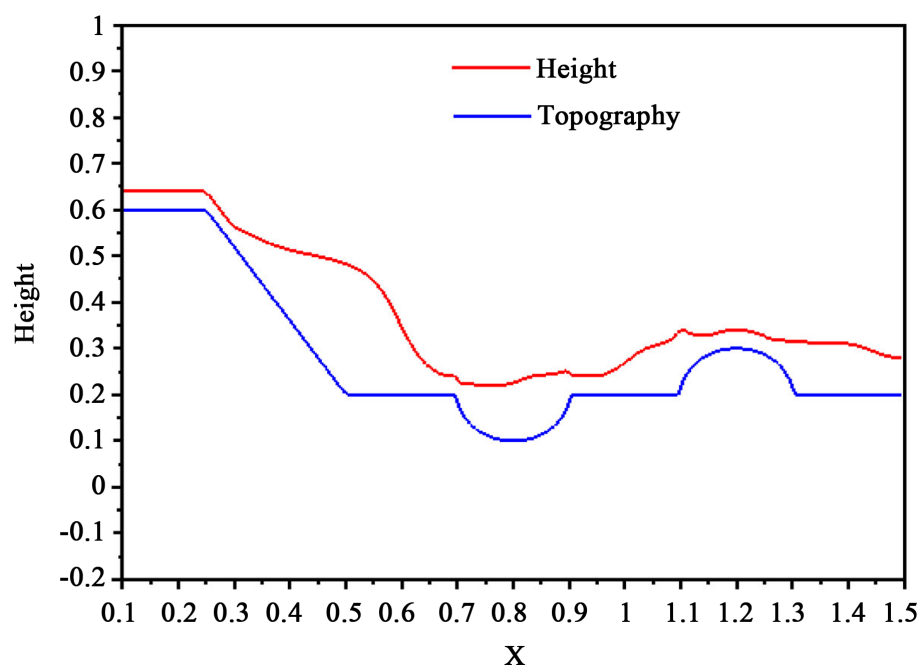

Figure 5. Water height at $t=0.017 \mathrm{~s}$.

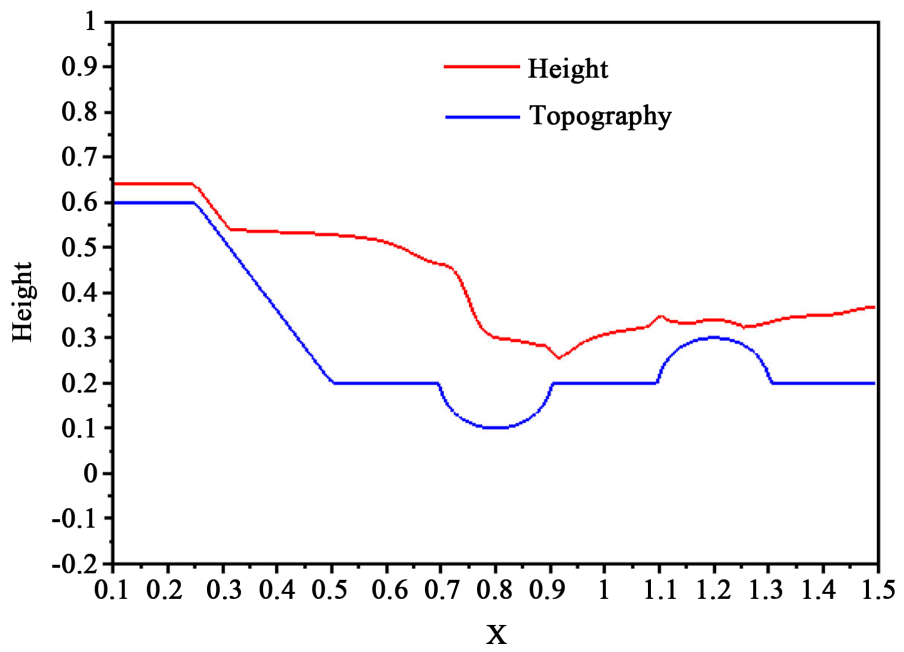

Figure 6. Water height at $t=0.026 \mathrm{~s}$. 


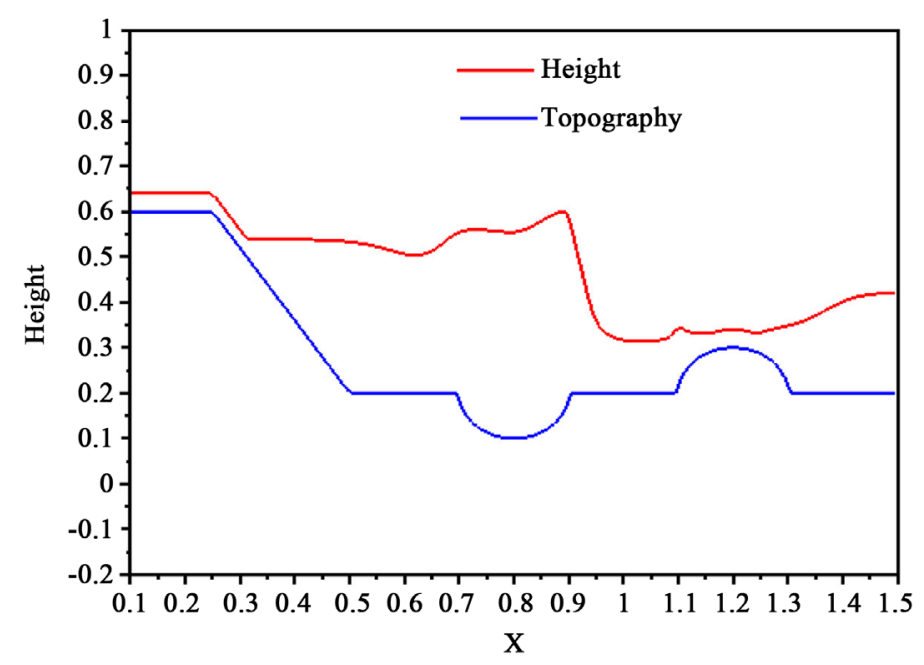

Figure 7. Water height at $t=0.036 \mathrm{~s}$.

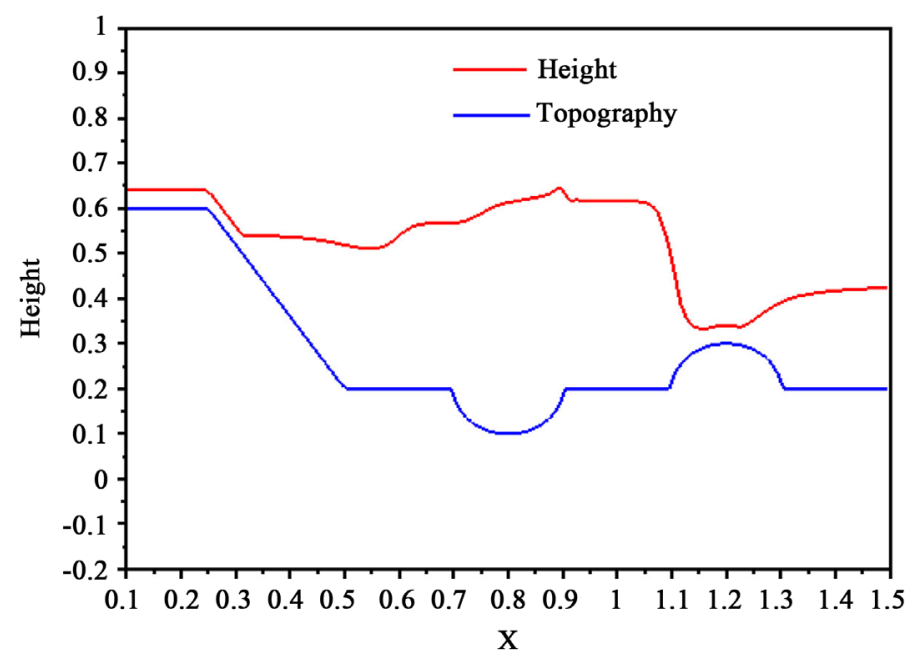

Figure 8. Water height at $t=0.046 \mathrm{~s}$.

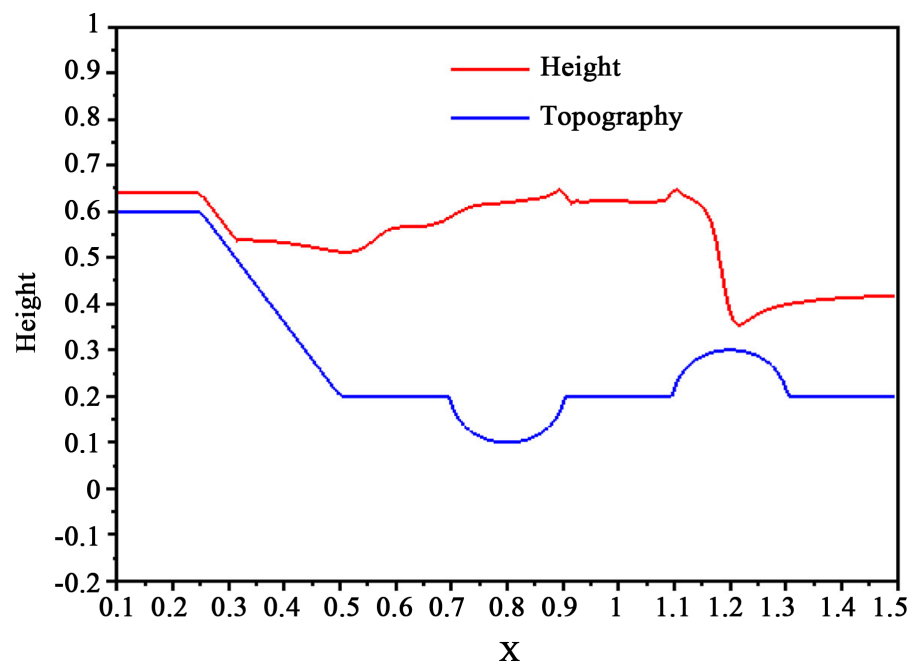

Figure 9. Water height at $t=0.051 \mathrm{~s}$. 


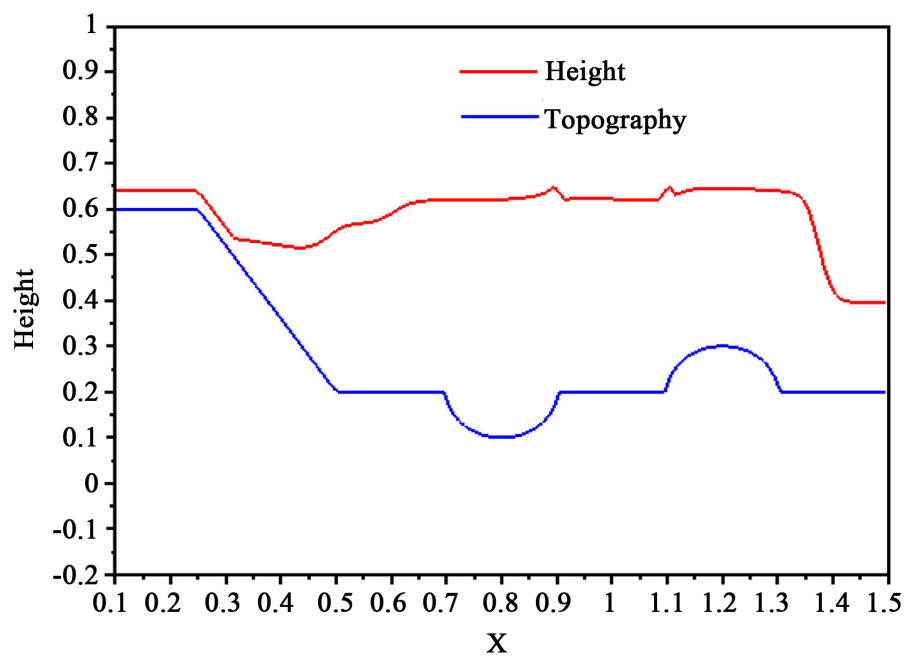

Figure 10. Water height at $t=0.061 \mathrm{~s}$.

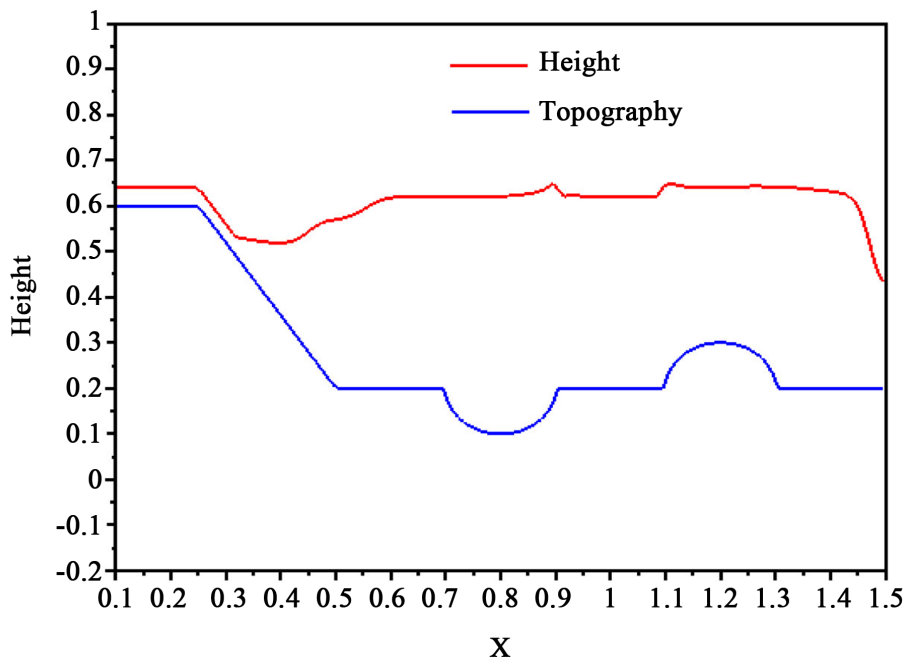

Figure 11. Water height at $t=0.066 \mathrm{~s}$.

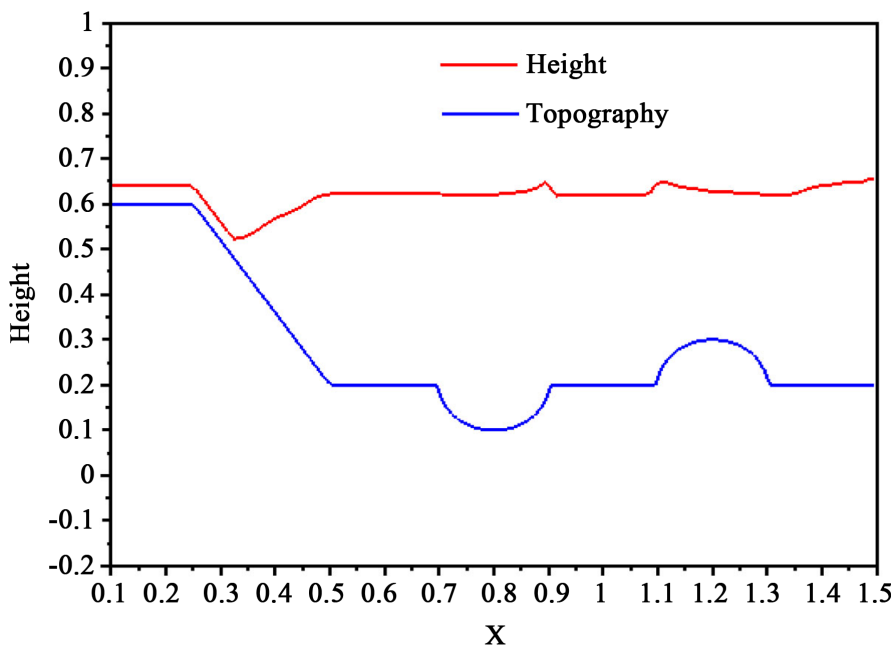

Figure 12. Water height at $t=0.075 \mathrm{~s}$. 


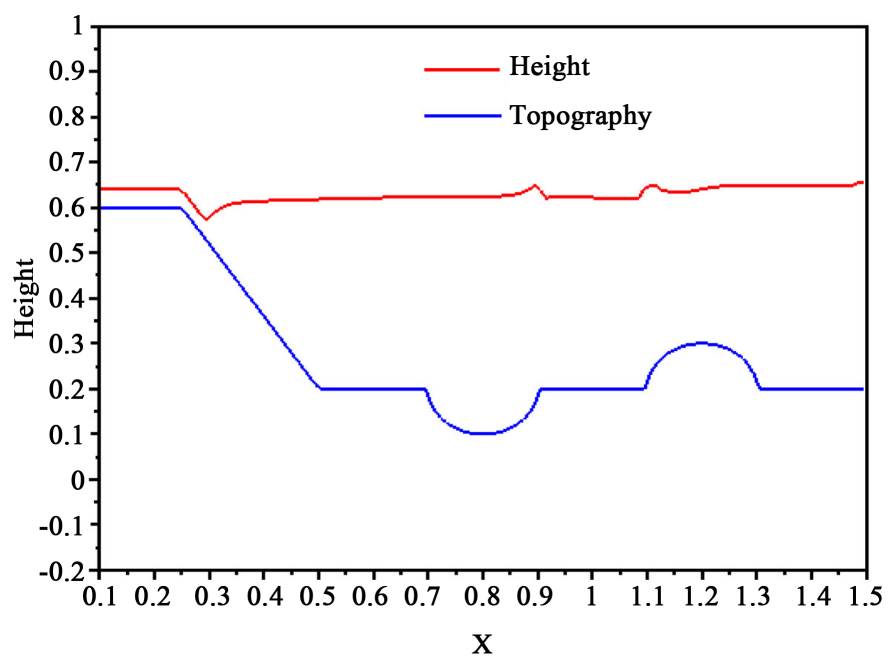

Figure 13. Water height at $t=0.090 \mathrm{~s}$.

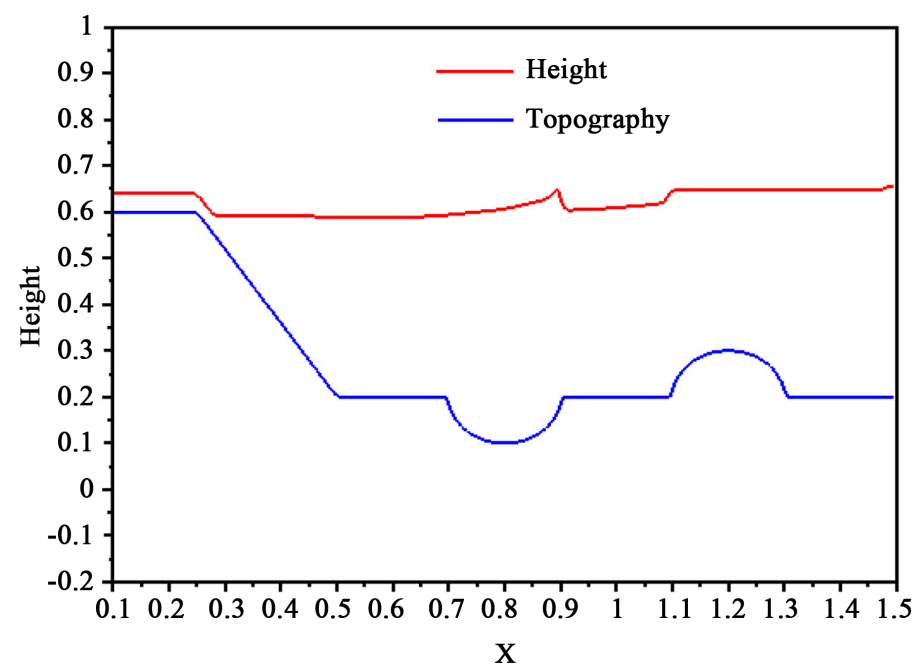

Figure 14. Water height at $t=0.11 \mathrm{~s}$.

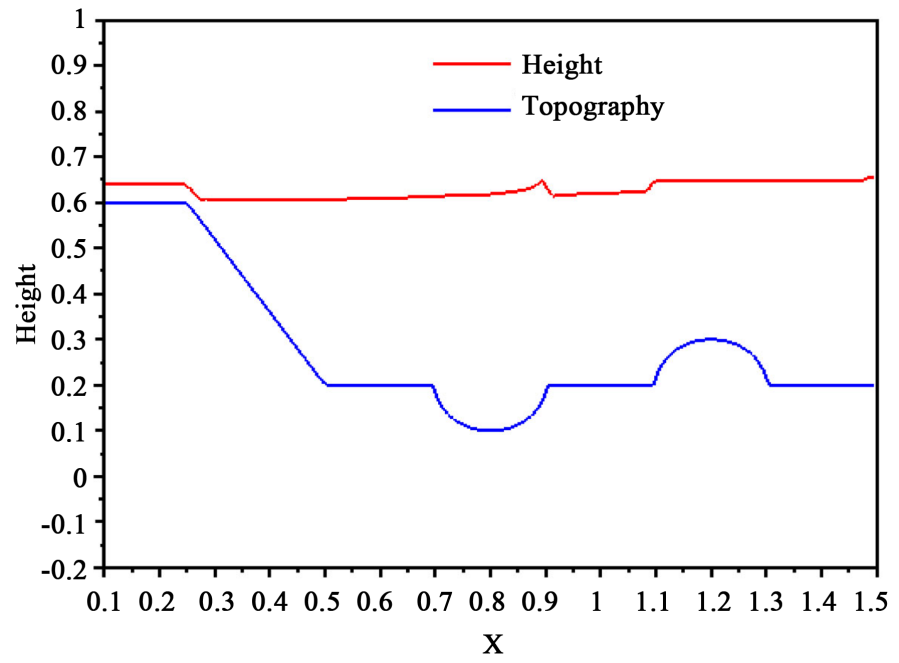

Figure 15. Water height at $t=0.19 \mathrm{~s}$. 


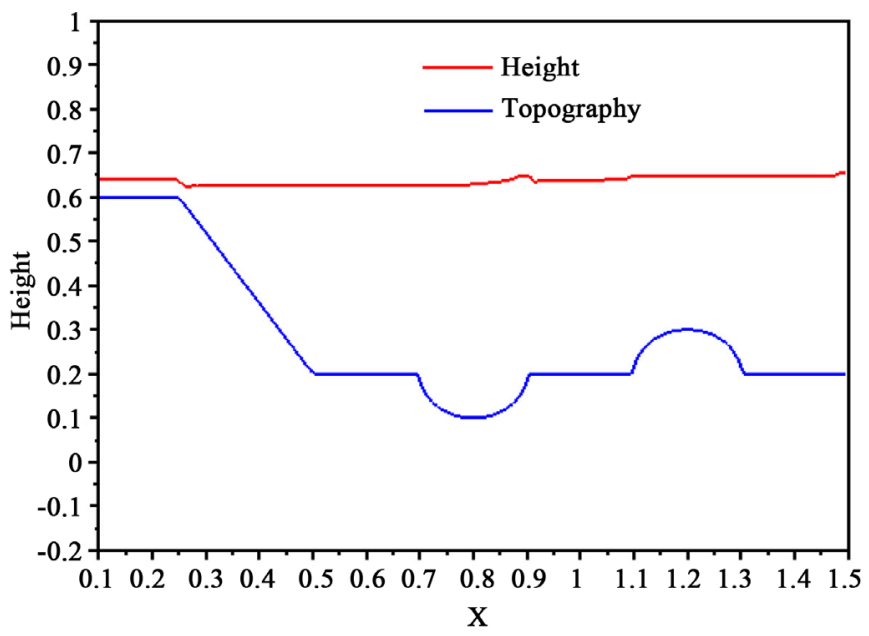

Figure 16. Water height at $t=0.58 \mathrm{~s}$.

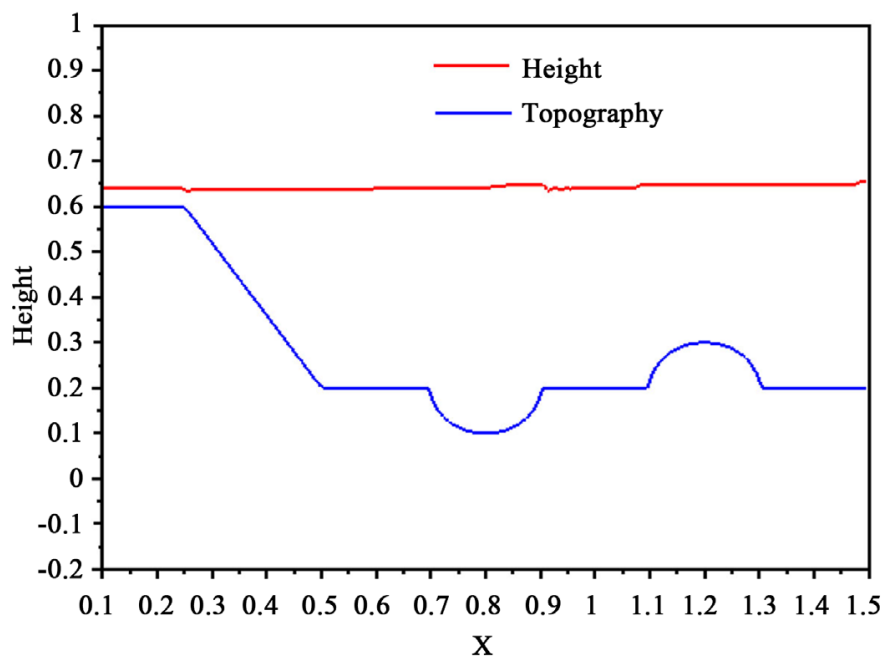

Figure 17. Water height at $t=0.97 \mathrm{~s}$.

\section{Conclusions}

The present work concerns the mathematical study of the flooding phenomenon in areas with variable topography. As a model we use the Saint-Venant equations, thus:

- We have proved the existence of a weak solution using a geometric argument.

- We point out through numerical simulations that the water first invades the hollow areas and then floods the areas with elevations.

- Our approach allows us to predict the necessary time for the water to invade a residential area, which is important for countries where constructions are made on not completely leveled grounds.

\section{Acknowledgements}

The authors thank the anonymous referees who provided valuable comments 
resulting in improvements in this paper. This work is supported by The World Academy of Science under grant: 18-047-RG/MATHS/AF/AC_I. The authors declare no conflicts of interest regarding the publication of this paper.

\section{Conflicts of Interest}

The authors declare no conflicts of interest regarding the publication of this paper.

\section{References}

[1] Delestre, O., Cordier, S., James, F. and Darbaux, F. (2008) Simulation of Rain-Water Overland-Flow. 12th International Conference on Hyperbolic Problems, College Park, June 2008, 537-546. https://doi.org/10.1090/psapm/067.2/2605249

[2] Delestre, O. and Marche, F. (2011) A Numerical scheme for a Viscours Shallow Water Model with Friction. Journal Scientific Computational, 48, 41-51.

[3] Benkhaldoun, F., Elmahi, I. and Seaid, M. (2006) Well-Balanced Finite Volume Schemes for Pollutant Transport on Unstructured Meshes. Journal of Computational Physics, 226, 180-203. https://doi.org/10.1016/j.jcp.2007.04.005

[4] Derakhshan, H. and Bistkonj, M.Z. (2013) A New Two Dimensional Model for Pollutant Transport in Ajichai River. Journal of Hydraulic Structures, 1, 44-54.

[5] Stoker, J.J. (1957) Water Waves: The mathematical Theory with Applications. Interscience, New York.

[6] Hervouet, J.-M. (2007) Hydrodynamics of Free Surface Flows Modelling with the Finite Element Method. John Wiley \& Sons, Hoboken.

[7] Edwige, G. and Raviart, P.A. (1991) Hyperbolic Systems of Conservation Laws. Series Mathematics and Applications, Ellipses, Paris.

[8] LeVeque, R.J. (2002) Finite Volume Methods for Hyperbolic Problems. Cambridge University Press, Cambridge, UK.

[9] Chen, G. and Noelle, S. (2015) A New Hydrostatic Reconstruction Scheme Motivated by the Wet-Dry Front. IGPM, RWTH Aachen University, Aachen.

[10] Edwige, G. and Raviart, P.A. (1996) Numerical Approximation of Hyperbolic Systems of Conservation Laws. Collection Applied Mathematical Sciences, 118. Springer-Verlag, New York.

[11] Duran, A., Liang, Q. and Marche, F. (2013) On the Well-Balanced Numerical Discretization of Shallow Water Equations on Instructured Meshes. Journal of Computational Physics, 235, 565-585. https://doi.org/10.1016/j.jcp.2012.10.033

[12] Audusse, E. and Bristeau. M.O. (2005) A Well-Balanced Positivity Preserving Second-Order Scheme for Shallow Water Flows on Unstructured Meshes. Journal of Computational Physics, 206, 311-333. https://doi.org/10.1016/j.jcp.2004.12.016

[13] Audusse, E., Bouchut, F., Bristeau, M.O., Klein, R. and Perthame, B. (2006) A Fast and Stable Well-Balanced Scheme with Hydrostatic Reconstruction for Shallow Water Flows. SIAM Journal on Scientific Computing, 25, 2050-2065. https://doi.org/10.1137/s1064827503431090 


\section{Notations}

The support of the function $f$ in $\Omega$ is $\operatorname{supp}(f)=\{x \in \Omega$ such as $f(x) \neq 0\}$. $C_{c}(\Omega)$ is the space of continuous functions with compact support in $\Omega$. $C^{k}(\Omega)$ is the space of functions k-th continuously differentiable in $\Omega$. $C^{\infty}(\Omega)=\bigcap_{k \in \mathbb{N}} C^{k}(\Omega)$.

$C_{c}^{k}(\Omega)=C^{k}(\Omega) \cap C_{c}(\Omega)$.

$L^{p}(\Omega)=\left\{f: \Omega \rightarrow \mathbb{R}, \int_{\Omega}|f(x)|^{p} \mathrm{~d} x<+\infty\right\}$.

$L^{\infty}(\Omega)=\{f: \Omega \rightarrow \mathbb{R}, \exists K \geq 0,|f(x)| \leq K$, a.e. $x \in \Omega\}$. 